\title{
Rectifying postures reconstructed from joint angles to meet constraints
}

\author{
Julian J. Faraway ${ }^{\mathrm{a}, *}$, Xudong Zhang ${ }^{\mathrm{b}}$, Don B. Chaffin ${ }^{\mathrm{c}}$ \\ ${ }^{a}$ Department of Statistics, University of Michigan, 1440 Mason Hall, 419 South State Street, Ann Arbor, MI 48109-1027, USA \\ ${ }^{\mathrm{b}}$ Department of Biomedical and Human Factors Engineering, Wright State University \\ ${ }^{\mathrm{c} C e n t e r ~ f o r ~ E r g o n o m i c s, ~ U n i v e r s i t y ~ o f ~ M i c h i g a n ~}$ \\ Received in final form 11 February 1999
}

\begin{abstract}
Postures are often described and modeled using angles between body segments rather than joint coordinates. Models can be used to predict these angles as a function of anthropometry and postural requirements. Postural representation, however, requires the joint coordinates. The use of conventional forward kinematics to derive joint coordinates from predicted angles may violate task constraints, such as the placement of a hand on a target or a foot on a pedal. Errors arise because the anthropometry or other motion characteristics of a subject, for which the prediction is to be made, may differ from the data from which the prediction model was derived. We describe how to rectify model-predicted postures to exactly satisfy such task constraints. We require that the model used for predicting the angles also produce estimates of the variation in these predictions. We show how to alter the initial angle predictions, with the amount of perturbation at each angle dependent on the accuracy of its estimation, so as to exactly satisfy the joint coordinate constraints. Finally, we show in an empirical example that this correction usually produces better overall predictions of posture than those obtained initially. (C) 1999 Elsevier Science Ltd. All rights reserved.
\end{abstract}

Keywords: Optimization; Postural constraints; Pseudoinverse

\section{Introduction}

In movement or posture modeling, it is often desirable to describe body motion in terms of angles formed by body segments rather than attempting to model the coordinates of the joints directly. Angles are more invariant to changes in body size which means the applicable range of angle-based models will be greater. They also are the primary variables in kinematic and biomechanical analysis using well-established methodologies (e.g. Greenwood, 1988). Further, the posture can be specified more compactly in terms of angles rather than joint coordinates. Ultimately, however, the angular description of posture needs to be converted to joint coordinates so that the posture can be rendered and observed in a global coordinate system perhaps used by CAD software.

The kinematic linkage representing the human body (or some portion thereof) may be geometrically constrained so that both ends have to be at specified locations. For example, we may wish to predict the posture of

\footnotetext{
* Corresponding author. Tel.: +734-763-5238; fax: +734-763-4676; e-mail: faraway@umich.edu.
}

a seated individual during a reach to an object. The posture must satisfy a seating constraint while placing the hand on the target at the end of the motion. For example, the authors conducted a study of reaching motions of drivers within an automobile. Motion capture techniques were used to record the coordinates of the joints of 38 subjects while they reached to 76 targets. The problem is to predict the posture of each new subject reaching to various targets. If the new subject's anthropometry and motion characteristics and the location of the new target do not match a record in the database, then we might consider constructing a model that will predict the desired posture given the subject and target information as inputs. For this construction, angle-based models are preferable for the reasons stated above, which led us to two different approaches in Faraway (1997) and Zhang (1997). Other such models can be found in Sepulveda et al. (1993) and Yamaguchi et al. (1995). These models allow the reconstruction of posture from predicted angles. The drawback of these models is that they do not guarantee that the reconstructed posture will have the hand, for instance, on the target. There are two reasons for the error. Firstly, the prediction models are not perfect so there will be some error in the angle 
predictions. Secondly, the lengths of the body segments may not be an explicit component of such models and so this variation will result in joint coordinate errors in the reconstruction. Ideally, an angle-based model should incorporate task endpoint constraints directly, but this would require a very complex model.

This article presents a coherent method for rectifying initial angle predictions to satisfy constraints on both ends of a kinematic linkage. This method will be useful when more accurate predictions are required. We believe the method is general and will work with a wide variety of angle prediction models.

\section{Methods}

We begin by representing a specific person as a collection of articulated linear rigid segments whose lengths are $1=\left(1_{1}, \ldots, 1_{n}\right)$, where $n$ is the number of links. Furthermore, suppose that the angles predicting a body posture are $\tilde{\theta}=\left(\widetilde{\theta}_{1}, \ldots, \widetilde{\theta}_{m}\right)$ where $m$ angles are sufficient to specify the posture. Suppose that the root of the kinematic linkage of body segments is the origin in our coordinate system. We can do this without loss of generality - this origin could be some point in the pelvis in the case of a seated operator. The target has coordinates $\mathbf{x}_{\mathbf{e}}$. Starting from the origin we can build the posture using $\tilde{\theta}$ and 1 . The end of the final segment is at $\mathbf{x}_{\mathrm{h}}$, (which we will call the hand for ease of reference, but it could be some other body part). The methodology to be presented will work equally well in two or three dimensions.

Ideally, the predictions $\tilde{\theta}$ should be constrained so that a match between $\mathbf{x}_{\mathrm{h}}$ and $\mathbf{x}_{\mathrm{e}}$ occurs, but such a prediction model would be highly nonlinear and complex. The model would require the use of 1 as an input, which goes against the original intention of modeling posture in terms of angles only. Furthermore, the regression model which predicts $\tilde{\theta}$ may use information such as age, gender or other human attributes, and may well allow the target $\mathbf{x}_{\mathrm{e}}$ to be varied continuously. It would be very difficult to ensure under these circumstances that the model will give a $\tilde{\theta}$ that puts the hand exactly on the desired target. The task now is to adjust the initial $\tilde{\theta}$ predictions to put the hand on the target while moving the rest of the posture as little as possible.

Suppose that the predictive distribution for $\theta$, the "true" angles, is multivariate normal, $N(\widetilde{\theta}, \Sigma)$. This means that $\tilde{\theta}$ is simply the most likely prediction, but that other predictions close to this value have some reasonable likelihood. $\Sigma$ is the covariance matrix for the prediction distribution. The form this matrix takes will depend on how the angular predictions are produced. Not all prediction methods will explicitly produce a multivariate normal predictive distribution, but it will usually be reasonable to assume just such an underlying distribution. If the predictions of the angles are made indepen- dently and are thought to be of equal accuracy, we may set $\Sigma=\sigma^{2} I$ where $I$ is an $m \times m$ identity matrix and $\sigma^{2}$ is the variance of prediction. It is not necessary to know the value of $\sigma^{2}$ for our method to work. In some cases, the predictions may be independent, but not of equal variance, in which case set $\Sigma=\operatorname{diag}\left(\sigma_{1}^{2}, \ldots, \sigma_{m}^{2}\right)$ where $\sigma_{i}^{2}$ is the variance of $\widetilde{\theta_{i}}$. Again it will only be necessary to know the relative and not the absolute values of the $\sigma_{i}^{2}$ 's. In many cases, the angle estimates will be correlated and if the form of this correlation is known or can be estimated, it should be used.

We now wish to find the most likely predicted angles $\tilde{\theta}$ subject to satisfying the constraint that the hand lies on the target. Since we are assuming a multivariate normal distribution for $\theta$, this is equivalent to finding the $\widetilde{\theta}$ that minimizes

$(\hat{\theta}-\tilde{\theta})^{\mathrm{T}} \Sigma^{-1}(\hat{\theta}-\tilde{\theta})$,

subject to the constraint

$f(\hat{\theta}, \mathbf{l})=\mathbf{x}_{\mathrm{e}}$.

The relationship between the hand position $\mathbf{x}_{\mathrm{h}}$ and the angles $\theta, \mathbf{x}_{\mathbf{h}}=f(\theta, \mathbf{I})$ can be derived in matrix forms using standard rigid body kinematics, and is typically a system of three equations for a 3D prediction. Differentiating this relationship results in

$\Delta \mathbf{x}_{\mathrm{h}}=\frac{\partial f}{\partial \theta} \Delta \theta$,

where $\partial f / \partial \theta$ is the Jacobian and will be abbreviated $J$. We can then solve the problem using the pseudoinverse (see Boullion and Odell, 1971). The solution is obtained by iterating, $i=1,2, \ldots$,

$\hat{\theta}_{(i)}=\hat{\theta}_{(i-1)}+\sum J^{\mathrm{T}}\left(J \Sigma J^{\mathrm{T}}\right)^{-1}\left(\mathbf{x}_{\mathrm{e}}-\hat{\mathbf{x}}_{(i-1)}\right)$,

where $\hat{\mathbf{x}}_{(i)}=f\left(\hat{\theta}_{(i)}, \mathbf{l}\right)$ and where $J$ is computed at each iteration using $\hat{\theta}_{(i-1)}$ and the initial conditions set $\hat{\theta}_{(0)}=\tilde{\theta}$. In our examples we needed no more than three iterations to produce acceptable results.

We tested the performance of our posture rectification method by using data from an automobile driver reaching for a target in the instrument panel area. We used a four segment linkage as shown in Fig. 1. To avoid dependence on a particular method of joint angle prediction, we perturbed the measured angles of an average male subject using normally distributed errors to produce simulated predicted angles. We then compared the predicted posture using these perturbed angles with the rectified predictions that put the hand precisely on the radio control. We now wish to see what the rectification does to the predictions of the elbow and shoulder locations.

The four segment linkage system had seven degrees of freedom, which were defined using Euler angles, to 


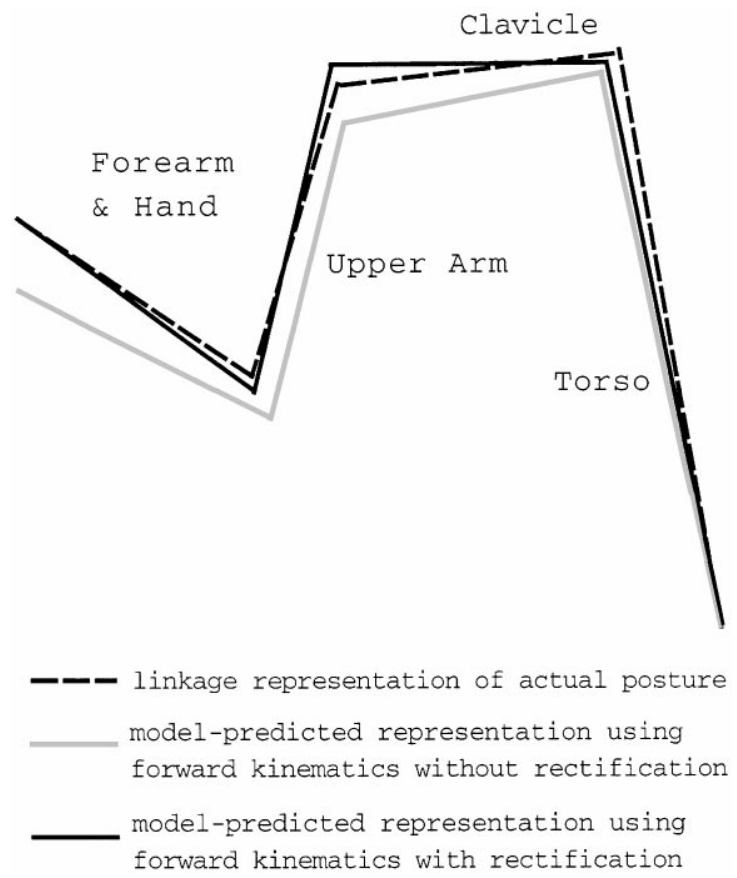

Fig. 1. A four-segment linkage representation of the upper body posture during seated reaching movement. This linkage is used to illustrate the proposed method that rectifies the predicted postures such that the hand reaches the intended target when the postures are reconstructed using forward kinematics. Seven degrees of freedom are embedded in the linkage: (1) torso flexion/ extension, (2) torso lateral bending, (3) clavicle rotation, (4) shoulder/extension, (5) shoulder adduction/abduction, (6) humeral rotation, and (7) elbow flexion/extension.

describe the kinematics of the linkage (see Zhang et al., 1998, for the exact definitions). A seated reaching posture of an average male subject in an automobile with his hand on the radio controls was measured to provide the $3 \mathrm{D}$ coordinates of the following joints: Anterior-superior iliac spine which is fixed, sternum, shoulder, elbow and hand (fixed wrist). The seven measured angles were then derived from the joint coordinates. We used three perturbation schemes to generate the simulated predicted angles. We have found in prior work that variability in repeated motions becomes greater as you move from the sternum to the shoulder to the elbow so we perturbed the angles associated with the sternum, shoulder and elbow in a ratio of $1: 2: 3$. Scheme $A$ : Low variance and no bias where the perturbation SDs are 1,2 and $3^{\circ}$, respectively. Scheme B: High variance and no bias where the perturbation SDs are 3, 6 and $9^{\circ}$ respectively. Scheme C: Low variance and bias where the perturbation SDs are 1, 2 and $3^{\circ}$ and the biases are 1,2 and $3^{\circ}$ respectively. Thus the perturbed angles were generated by adding the specified bias to the true angle and then adding a zero-mean randomly generated normally distributed term with the specified variance. One hundred trials were used for each scheme.

The joint angles were then calculated using both a conventional forward kinematics method (without rectifica- tion) and using the proposed rectification scheme. We computed the distance of the unrectified joint locations to the true joint locations. We report below the percentage reduction in these distances due to rectification.

\section{Results}

When the angle predictions are unbiased, rectification will generally improve the joint center predictions, but when there is some bias in the angle predictions, rectification may worsen the joint center prediction at the sternum and shoulder while improving them at the elbow and hand. The median percentage reduction in error for each joint location, shown in Table 1, is positive for the variance only schemes $\mathrm{A}$ and $\mathrm{B}$, but is negative at the sternum and shoulder for the variance and bias scheme C. The median errors in $\mathrm{mm}$ before and after rectification are shown in Table 2. We observe that the rectification is preferred except at the sternum and shoulder for C, although even in this scheme the total error is reduced. We deliberately included an example where rectification made some parts of the posture prediction worse. In other biased angle prediction schemes we tried, rectification improved the posture prediction at every joint.

\section{Discussion}

We developed this method because we could find no solution to the problem of correcting predicted postures derived from angle-based models to satisfy endpoint constraints in the literature. The method will work with any angle-based prediction model where the user has some knowledge of the relative variability in these predictions.

Table 1

Median of the percent improvement in correction

\begin{tabular}{lcccc}
\hline Scheme & Sternum & Shoulder & Elbow & Hand \\
\hline A & 19 & 24 & 50 & 100 \\
B & 11 & 19 & 53 & 100 \\
C & -23 & -36 & 32 & 100 \\
\hline
\end{tabular}

Table 2

Median error $(\mathrm{mm})$ before and after correction

\begin{tabular}{llrccc}
\hline & & Sternum & Shoulder & Elbow & Hand \\
\hline Scheme A & Before & 6.2 & 8.8 & 16.0 & 30.5 \\
& After & 4.5 & 5.5 & 7.6 & 0 \\
Scheme B & Before & 18.4 & 24.0 & 51.7 & 96.8 \\
& After & 17.3 & 19.5 & 23.6 & 0 \\
Scheme C & Before & 8.9 & 11.4 & 19.3 & 50.0 \\
& After & 11.6 & 16.3 & 14.0 & 0 \\
\hline
\end{tabular}


We require that the kinematic linkage only be constrained by the endpoints. The method could not directly accommodate other constraints such as those imposed by the range of mobility at various joints. The method also requires the computation of the Jacobian $J$, which although straightforward in theory, can be tiresome in practice, especially if there are large numbers of segments in the linkage.

\section{Acknowledgements}

The authors acknowledge the support of the Chrysler Corporation Challenge fund and Dr. Deborah Thompson in particular.

\section{References}

Boullion, T.L., Odell, P.L., 1971. Generalized Inverse Matrices. Wiley, New York.
Faraway, J.J., 1997. Regression analysis for a functional response. Technometrics 39, 254-261.

Flash, T., Hogan, N., 1985. The coordination of arm movement: an experimentally confirmed mathematical model. Journal of Neuroscience 7, 1688-1703.

Greenwood, D.T., 1988. Principles of Dynamics, 2nd ed. Prentice-Hall, Englewood Cliffs, NJ.

Sepulveda, F., Wells, D.M., Vaughan, C.L., 1993. A neural network representation of electromyography and joint dynamics in human gait. Journal of Biomechanics 26, 101-109.

Yamaguchi, G.T., Moran, D.W., Si, J., 1995. A computationally efficient method for solving the redundant problem in biomechanics. Journal of Biomechanics 28, 999-1005.

Zhang, X., 1997. The development of a three-dimensional dynamic posture prediction model for seated operator motion simulation. Doctoral dissertation, The University of Michigan, Ann Arbor.

Zhang, X., Kuo, A.D., Chaffin, D.B., 1998. Optimization-based differential kinematic modeling exhibits a velocity-control strategy for dynamic posture determination in seated reaching movements. Journal of Biomechanics 31, 1035-1042. 